\title{
Throughput and Handover Latency Evaluation for Multicast Proxy Mobile IPV6
}

\author{
Azana Hafizah Mohd Aman, Aisha-Hassan A. Hashim*, Huda Adibah Mohd Ramli \\ Kulliyyah of Engineering, International Islamic University Malaysia, \\ Jalan Gombak 53100, Kuala Lumpur, Malaysia \\ ${ }^{*}$ Corresponding author, e-mail: aisha@iium.edu.my
}

\begin{abstract}
The objective of this paper is to present performance analysis of a new enhanced mobile multicast network mobility management scheme. The initial developed network mobility management called Proxy Mobile IPv6 (PMIPV6) is based on unicast network support. This paper enabled multicast support in network mobility management and named it as MPMIPV6. Additionally this enhancement also provides better network performance with the new context transfer operations and fast reroute operations. In brief, this paper also describes other current mobile multicast schemes. The new scheme is evaluated using mathematical analysis and NS3.19 simulator. Theoretically this scheme reduces service recovery time, total signalling cost, handover latency, and packet loss for multicast communication. However for this paper, the analysed parameters are throughput and handover latency. Both mathematical and simulation results exhibit better network performance for multicast environment compared to the standard benchmark scheme.
\end{abstract}

Keywords: multicast, network mobility, mobile multicast, network mobility management

\section{Introduction}

This paper presents a new scheme for network mobility management. It is an improvement of operations by enhancing multicast mobility using Context Transfer (CT) [1] and Multicast Only Fast Reroute [2]. With this new enhanced scheme multicast is successfully enabled with better content delivery. The multicast contents are transferred in advance. The contents are carried out using two different routes, in order to ensure the delivery of the packets to the mobile node. This new operations provide better handover performance and at the same time enhance the mobile multicast. The design goals are to minimize the time needed to reestablish the multicast service as much as possible and to minimize the possibility of losing multicast packets.

PMIPv6 [3] is an enhancement to the basic IP mobility for IPv6 hosts that are specified in MIPv6 [4-5]. PMIPv6 is a network mobility management to ease the IP mobility problems. By extending MIPv6 signaling messages between a network node and a home agent (HA), PMIPv6 makes it possible to provide mobility for IPv6 nodes without requiring host involvement. A proxy mobility agent in the network does the mobility management on behalf of the mobile node attached to the network.

MLDv2 [6] is a component of the IPv6. MLDv2 is used to discover multicast listeners by IPv6 routers and to determine group membership on a network segment, also known as a link or subnet on a directly attached link. Multicast traffic is processed by multiple hosts. Multicast traffic is sent and processed by host that belong to the multicast group receive in the group's reserved address.

CTP [1] is used to transfer context of services. Mobile nodes (MN) with context transfers support allow applications running on it to operate under minimal disturbance. Context Transfers Protocol is applied to support optimized handover. It leads to optimized mobile node performance in mobile multicast environment. CTP reduces latency, packet losses and minimizing the re-establishment of services from scratch.

MFR [2] is basically a mechanism to minimizing packet loss issue. MFR is a self-detect network failure. MFR defines a primary path and a secondary path. Data packets are sent over both paths, the primary and secondary paths. But only packets from the primary are accepted 
and forwarded while the packets from the secondary are discarded. When a failure is detected on the path to the primary, the repair occurs by changing the secondary into the primary and the primary into the secondary. Since the repair is local, it is fast hence improving convergence times in the event of node or link failures on the path to the primary.

\section{Research Method}

This paper evaluates the enhanced scheme using mathemathical and simulation methods. Figure 1 and Figure 2 shows the process involves in mobile multicast communication. Figure 1 defines the enhanced scheme signaling call flow for intra domain multicast network. While Figure 2 defines the enhanced scheme signaling call flow in inter domain multicast network.

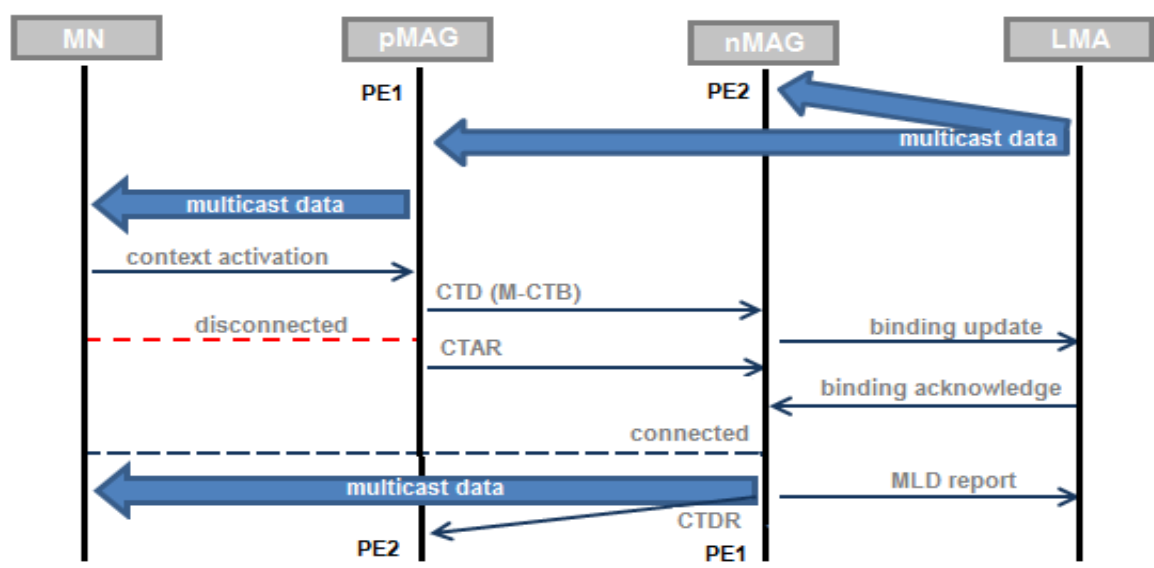

Figure 1. MPMIPv6 with CT-MFR intra domain handover signalling call flow

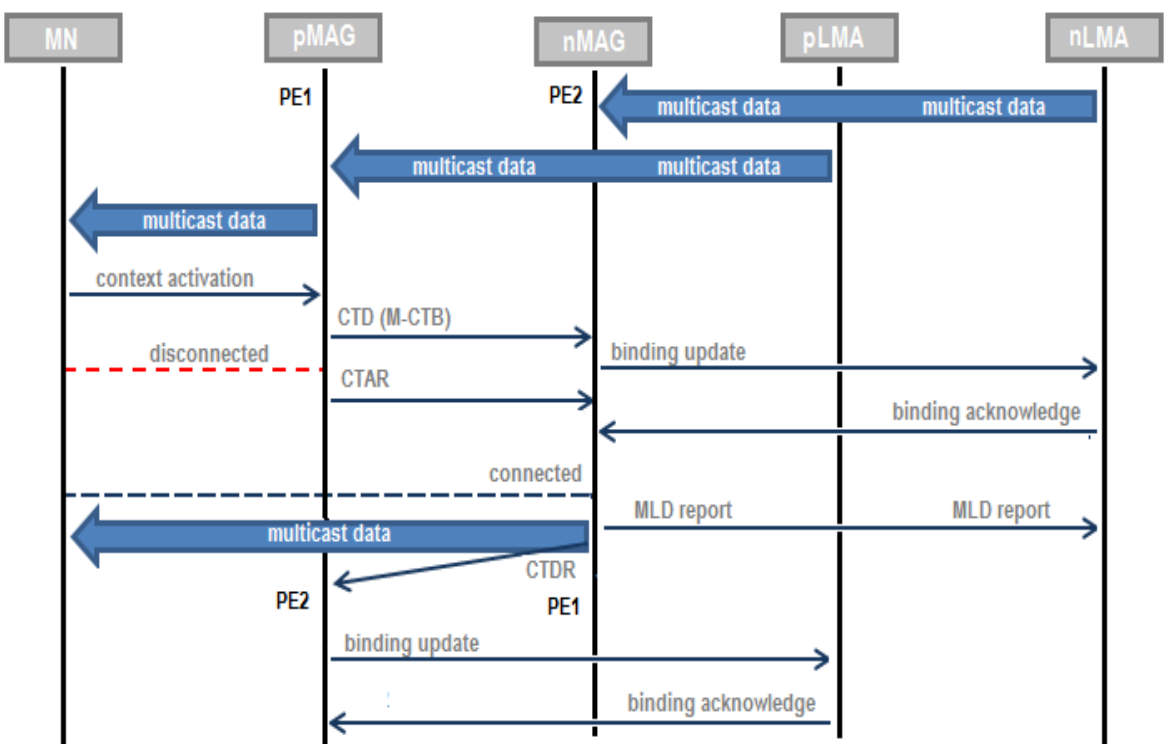

Figure 2. MPMIPv6 with CT-MFR inter domain handover signalling call flow

Figure 3 explains the enhanced scheme flow chart in intra domain multicast network. Figure 4 explains the enhanced scheme flow chart in inter domain multicast network. 


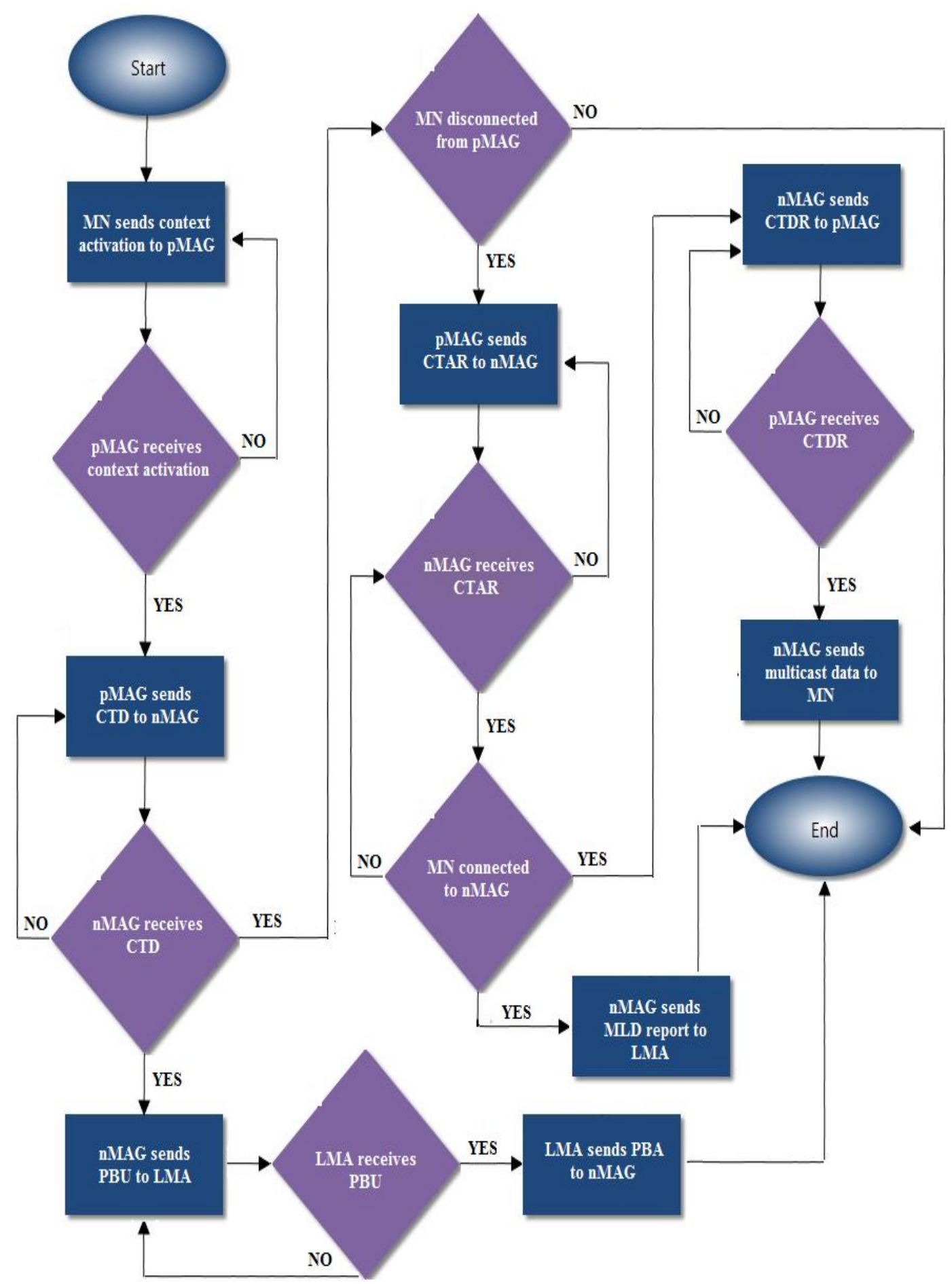

Figure 3. Intra domain handover flow chart for the enhanced scheme 


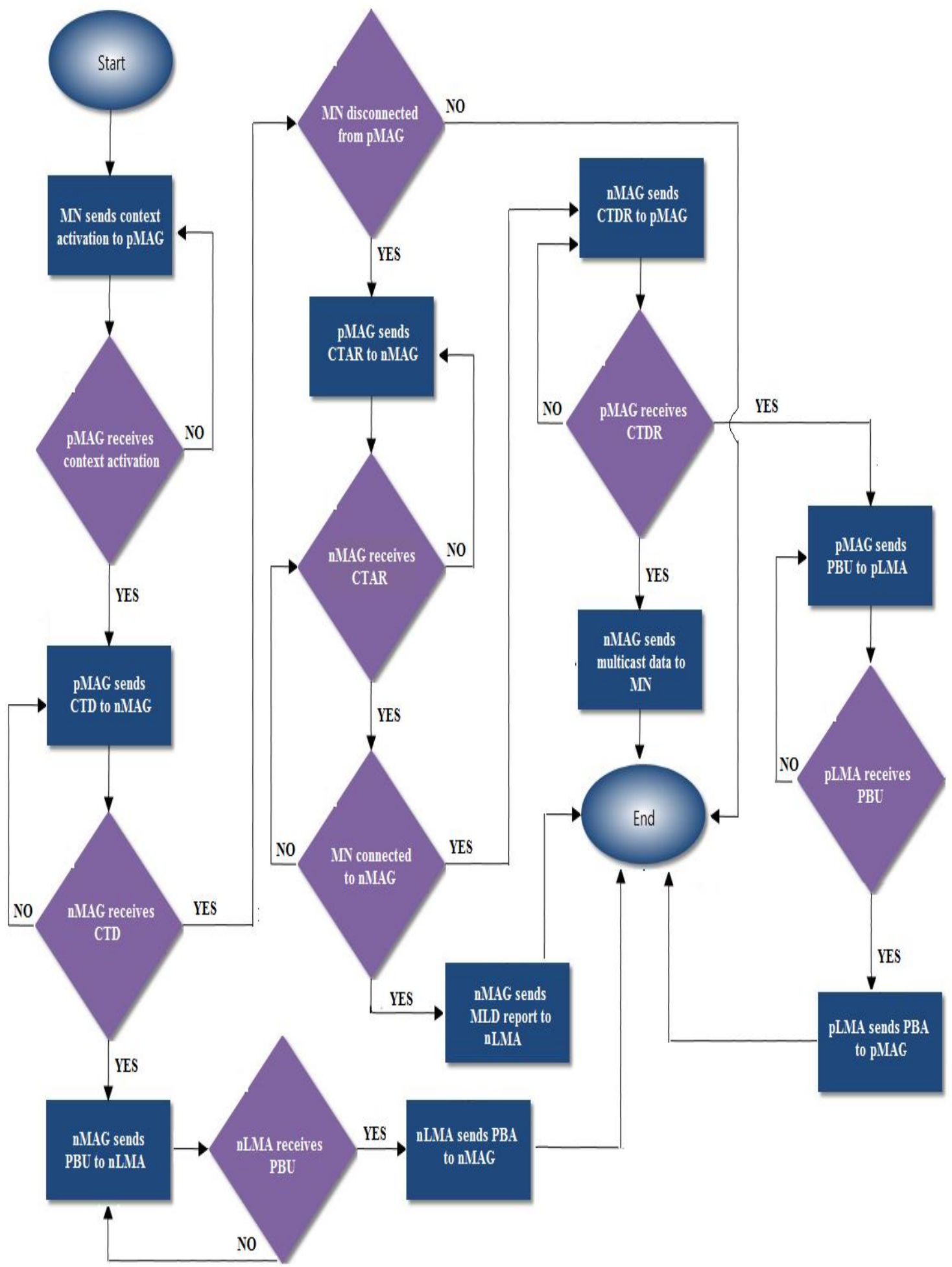

Figure 4. Inter domain handover flow chart for the enhanced scheme

\section{Results and Analysis}

In data transmission, network throughput is the amount of data moved successfully from one place to another in a given time period [7]. It is the number of messages successfully delivered per unit time. In this paper the throughput is extracted from the simulation process. 
Figure 5 illustrates the UDP throughput with respect to simulation time in second. It can be seen from the figure that when time increases, the throughput for both schemes decreases as the mobile node speed increases. The throughput for the benchmark is lower than the proposed scheme and keeps getting lower with higher decrease rate than the proposed scheme.

At simulation time 500 seconds, the proposed scheme performed more than 50\% compared to the benchmark. The drop of throughput faced by the benchmark is due to the changes of MN which is from old location to new location. As for the proposed scheme there is hardly any significant drop of throughput. This is due to the fact that when the time increases, the load time of the multicast session increases largely resulting long access time and congestion. In the proposed scheme, the MNs do not involve with the registration processes therefore resulted in a steady throughput increment as number of packets increases accordingly with simulation time. In the proposed scheme, the throughput efficiency is improved due to the benefit of CT-MFR predictive approach.

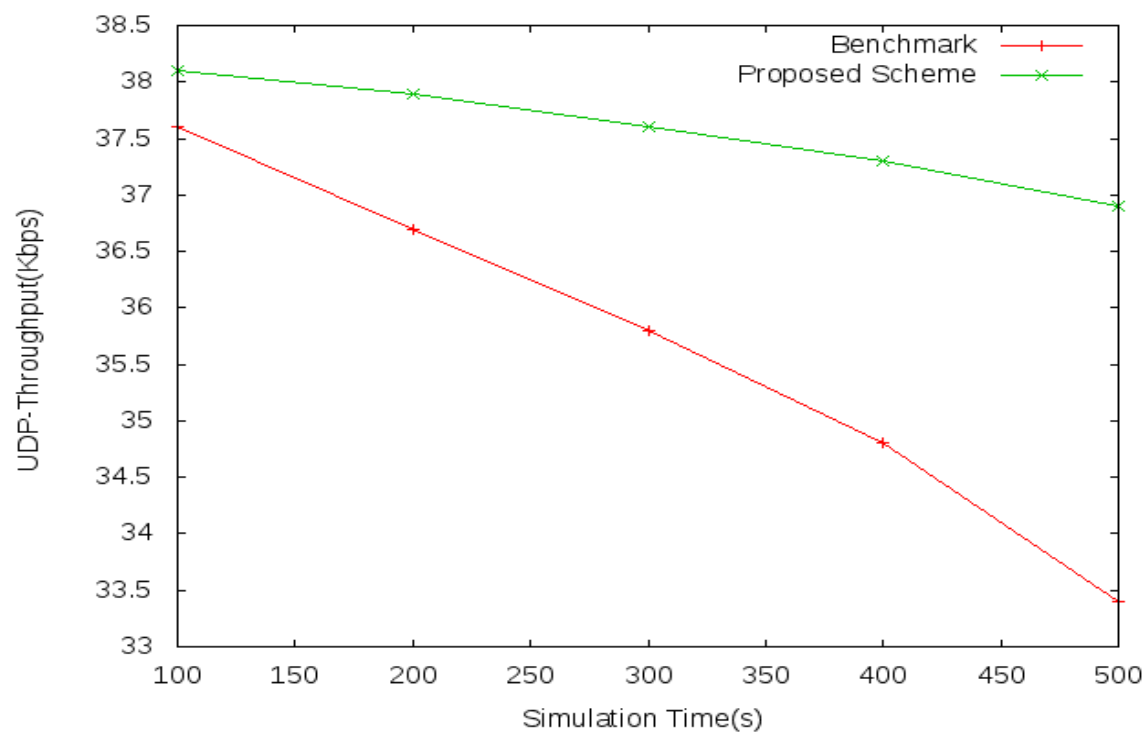

Figure 5. UDP throughput versus simulation time (s)

As indicated in Figure 6 the handover latency simulation result for the proposed scheme is $0.04 \mathrm{~s}$. The simulation result is nearly close to the mathematical results which is $0.07 \mathrm{~s}$. The range of handover latency value is between $0.04 \mathrm{~s}$ to $0.17 \mathrm{~s}$ as the simulation time increased.

The handover latency is defined as the time needed for the MN to change its point of attachment from one network connection to another [8]. Let $y$ denoted as handover latency, Ils as link switching delay, IRD denoted as router discovery delay. Table 1 summarizes the parameters for $\mathrm{ym}, \mathrm{\gamma c}$, and $\mathrm{ycmfr}[8]$.

Table 1 Parameters for Handover Latency

\begin{tabular}{llc}
\hline \multicolumn{1}{c}{ Parameter } & \multicolumn{1}{c}{ Description } & Value $(\mathrm{ms})$ \\
\hline$I_{\text {PMAGNMA }}$ & the time interval between PMAG and nMAG & 10 \\
$I_{L M A n M A G}$ & the time interval between LMA and nMAG & 10 \\
$I_{\text {MNnMAG }}$ & the time interval between MN and nMAG & 12 \\
$I_{\text {RD }}$ & router discovery delay & 10 \\
\hline
\end{tabular}

The handover latency for each solution is defined as below:

The handover latency for Multicast PMIPv6, $\mathrm{m}$, is expressed in equation 1:

$\mathrm{m}=I_{R D^{+}}+4 I_{M N n M A G}+3 I_{L M A n M A G}$ 
In case of Multicast PMIPv6 with CT, the handover latency, $\mathrm{c}$, is described in equation 2:

$$
\mathrm{c}=2 I_{\text {pMAGnMAG }}+3 I_{\text {LMAnMAG }}
$$
equation 3:

As for the Multicast PMIPv6 with CT-MFR, the handover latency, cmfr is, shown in

$$
\mathrm{cmfr}=I_{\text {PMAGNMAG }}+I_{L M A n M A G}
$$

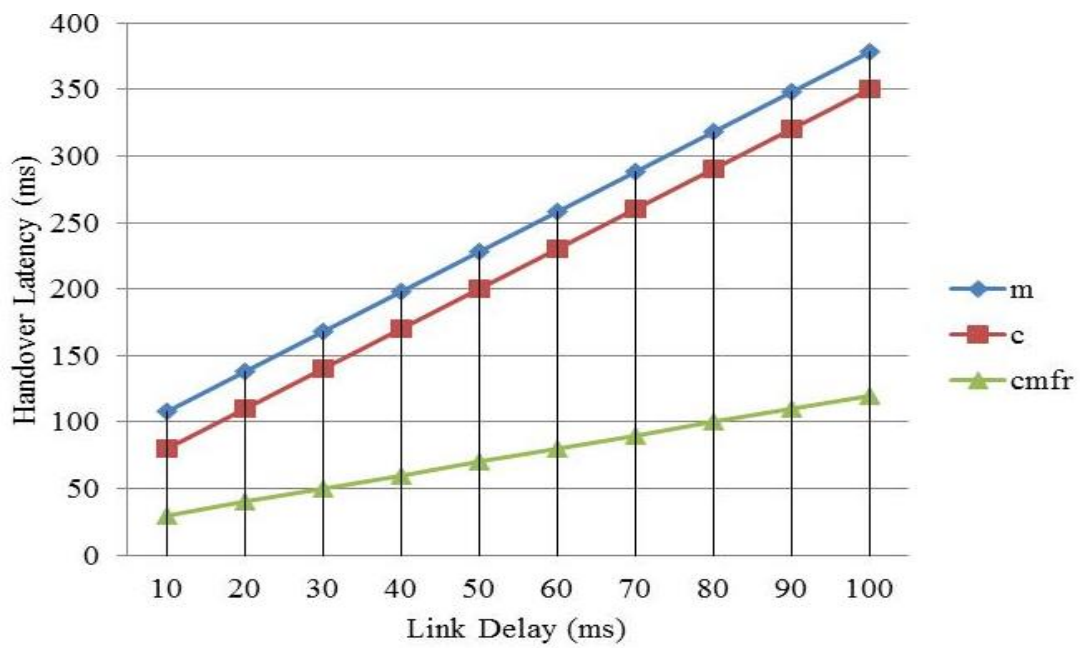

Figure 6. Handover latency (ms) versus link delay (ms)

\section{Conclusion}

A new scheme in mobility management protocol that integrates mobility with multicast is introduced. A new scheme that enhances mobile multicast with context transfer and multicast fast reroute is introduced. Multicast enabled PMIPv6 with CT-MFR improves the handover latency of $63 \%$ better than the benchmark PMIPv6. Multicast enabled PMIPv6 with CT-MFR presents throughput by total of $25 \%$ from the benchmarked scheme. Even though this work has successfully shown improvement in reducing handover latency, and throughput, further improvement is always welcome. As a future work, it is recommended to do real time simulation scenarios using hardware equipment such as mobile stations and wireless network devices.

\section{References}

[1] Loughney, M Nakhjiri, C Perkins, R Koodli. Context Transfer Protocol. RFC 4067. 2005.

[2] A Karan, C Filsfils, IJ Wijnands, B Decraene. Multicast Only Fasr Reroute (MoFRR). RFC 7431, 2015

[3] Gundavelli S, Ed Leung, K Devarapalli, V Chowdhury K, B Patil. Proxy Mobile IPv6 (PMIPv6). RFC 5213. 2008.

[4] Johnson D, Perkins C, J Arkko. Mobility support in IPv6. RFC 3775. 2004.

[5] Ismat Aldmour, Thair Al-Dala'in, Lelyzar Siregar, Rahmat Budiarto. SeamSAR: Seamless, Secure and Robust Handover Model for Mobile IPTV Network Using Enhanced FMIPv6. International Journal of Electrical and Computer Engineering (IJECE). April 2015; 5(2): 371-378.

[6] R Vida, L Costa. Multicast Listener Discovery Version 2 (MLDv2) for IPv6. RFC 3810. 2004.

[7] Farouk Abdul Jalin, Raed Alsaqour. A Simulation Study of Proxy Mobile IPV6 (PMIPV6) Protocol. Journal of Engineering and Applied Sciences. April 2016; 11(7): 4701-4706.

[8] Adnan J Jabir, S Shamala, Z Zuriati. A New Strategy for Signalling Overhead Reduction in the Proxy Mobile IPv6 Protocol. Am. J. of Applied Sci. 2012; 9(4): 535-541. 